\title{
Elevated c-myc Expression in Childhood Medulloblastomas
}

\author{
DOUGLAS N. MACGREGOR AND EDWARD B. ZIFF \\ Department of Pediatrics. Department of Biochemistry, and Kaplan Cancer Cenier. New York University \\ Medical Center, New York. New York 10016
}

\begin{abstract}
Medulloblastoma is a rare brain tumor usually occurring in late childhood or early adolescence. Little is known regarding the cell of origin or cellular events leading to its malignant transformation. We have studjed the expression of developmentally regulated mRNA in tumor samples by the Northern hybridization assay to determine a relative stage at which a block to further differentiation occurs. In a series of five medulloblastoma tumors analyzed, only one of eight markers for cellular differentiation, the glial fibrillary acidic protein mRNA, was expressed at significant levels. Interestingly, three of six medulloblastoma tumor samples were found to have elevated levels of c-myc mRNA. In one of these tumors, we have found evidence of mutation of the c-myc protooncogene. We discuss possible mechanisms of c-myc activation in medulloblastoma tumors. (Pediatr Res 28: 63-68, 1990)
\end{abstract}

\section{Abbreviations}

GFAP, glial fibrillary acidic protein PCR, polymerase chain reaction

Medulloblastoma brain tumors occur primarily in the pediatric population with $80 \%$ of all cases diagnosed before age 15 y (1). The cell of origin remains unknown, although some workers (2) suggest that the tumor arises from external granule cells, which are known to undergo postnatal mitosis and migration before their final differentiation. Although the tumor is statistically rare, it is the single most common brain tumor occurring in childhood (1). Despite significant improvements in multimodality therapy, including aggressive neurosurgical techniques, multiagent chemotherapy, and radiation therapy, only approximately $50 \%$ of children with this disease are cured (3). Within this group, there is a high incidence of serious neurologic sequelae.

Recently, molecular events have been described that may contribute to the development of CNS tumors. For example, the serum growth factor PDGF, which is the protein product of the cellular gene c-sis, has been found to be secreted by $N$-ethyl $N$ nitrosourea-induced glial brain tumors (4). Similarly, Liberman et al. (5) have reported amplification, enhanced expression, and possible rearrangement of the c-erb $\mathbf{B}$ gene in glial brain tumors. Of more specific interest to medulloblastomas, tumors of neural origin (2), is the family of myc genes. The oncogene $N$-myc was

Received June 30, 1989; accepted March 2, 1990.

Comespondence: Douglas N. MacGregor, M.D., Department of Biochernistny, New York University Medical Center, 550 First Avenue, New York, NY 10016

Supported by the Children's Cancer Fund no. 64-1-610l of the Department of Pediatrics, New York Unjversity Medical Center, the American Cancer Society Institutional Grant IN-14-30; the Department of Environmental Medicine of the New York University Medical Center (Grant ES00136); and NiH Grant IKIICA01419-01. first described (6) in tumor tissue arising from the peripheral nervous system, i.e. neuroblastomas. Evidence presented in this work links overexpression of the related protooncogene c-myc to medulloblastomas, tumors that arise from the CNS. Although the precise function of the c-myc gene product, a nuclear phosphoprotein (7), has not been defined, expression of the myc family may play a role in making cells competent to respond to signals that induce differentiation or proliferation (8). Enhanced c-myc gene expression has been found in a variety of tumors and cell lines $(9-12)$, which favors a link between c-myc expression and cellular proliferation. Several studies also support a role for $c-m y c$ in blocking differentiation. Constitutive expression of cmyc can block mouse erythroleukemia cell (13) and PCI 2 pheochromocytoma cell (14) differentiation in vitro and maintain the proliferative state of these cells in the presence of differentiation inducers.

In our report, we have assayed for molecular evidence of the extent of differentiation of five medulloblastoma tumors to identify a relative stage at which differentiation is blocked. mRNA isolated from tumor samples was screened with a panel of radiolabeled $\mathrm{cDNA}$ probes. These $\mathrm{CDNA}$ probes were selected on the basis that they correspond to functions expressed within the CNS at different stages of neuronal or glial development. From our panel of CDNA probes, only that for GFAP, an intermediate filament protein, was expressed at levels detectable by Northern assay in the tumor RNA. In three of six medulloblastoma tumors analyzed for c-myc expression, we found elevated levels of $c-m y c$ mRNA. Moreover, we found that GFAP mRNA expression appeared to be inversely correlated with $c$ myc mRNA levels in six of six informative tumors.

\section{MATERIALS AND METHODS}

Tissue samples. After approval by the Institutional Review Board of the New York University Medical Center, tumor samples were collected directly from the operating room and placed immediately in liquid nitrogen.

DNA probes. The following cDNA probes were used in this study: pGmycScrFI, a 72-bp subclone spanning the 3' end of the human c-myc lst exon; pmycX-PstI, a $1.1-\mathrm{kb}$ human c-myc subclone that includes $133 \mathrm{bp}$ of the $5^{\prime}$ end of the 2 nd exon; and pmyc 413-R/C, a 1.3-kb human c-myc 3rd exon subclone. The c-myc probes were kindly provided by Ethel Cesarman and Riccardo Dalla-Favera (15). A 1-kb $N$-myc subclone, pNb-1, was obtained from the American Type Culture Collections, Rockville, MD (41011). NF68 a 1.2-kb subclone of the murine 68000 mol wt neurofilament protein, was kindly provided by Lewis and Cowan (16). Plasmid Gl, a 2.5 -kb mouse subclone of GFAP was also provided by S.A. Lewis and N.J. Cowan (17). pSPT, a 1.8$\mathrm{kb}$ subclone of bovine chromogranin $\mathrm{A}$, was kindly provided by M. L. Grimes (18). Int- $I_{\mathrm{Es}}$, a 520 -bp subclone of mouse int-1, was kindly provided by D. G. Wilkinson (19). pM1B1, a 1.7-kb human subclone (H.1) for the homeobox protein Hox 2.1, was kindly provided by Craig Hauser (20). Clone 73 , which contains 
the cDNA for the rat neurofilament protein peripherin, was described by Leonard et al. (21). A 580-bp subclone of the murine neural cell adhesion molecule (N-CAM) mRNA, p1.3, was kindly provided by Christo Goridis (22). pHFBA-1, a 2.0-kb subclone of the human $\beta$-actin gene, was kindly provided by L. Kedes (23).

Isolation of RNA. Total cellular RNA was extracted by the guanidinium/cesium chloride method (24) from tumor samples frozen in liquid nitrogen. Total cellular RNA from HeLa cells, a cell line that is derived from a human cervical carcinoma and that expresses high levels of c-myc mRNA, was a gift from Anna Velcich (Montefiore Hospital, Bronx, NY).

Northem blot analysis. Northern analysis of $20-\mu \mathrm{g}$ aliquots of total cellular RNA was carried out as described (25) using fragments from plasmid subclones labeled by the method of Feinberg and Vogelstein (26).

Ribonuclease protection assay. The ribonuclease protection assay was performed by a modification of the procedure described by Winter et al. (27). For the synthesis of long RNA transcripts $(>1000 \mathrm{bp}$ ), cold cytidine 5 '-triphosphate was added to the reaction mixture to a final concentration of $10-20 \mu \mathrm{mol}$. Unincorporated label was removed by centrifugation through $\mathrm{G}$ 50 spin columns. Five $\times 10^{5} \mathrm{cpm}$ of the probe were coprecipitated with $20 \mu \mathrm{g}$ of RNA and then resuspended in hybridization buffer.

Isolation of DNA and Southern blot analysis. DNA was isolated (28) from medulloblastoma tumor tissue frozen in liquid nitrogen. Ten $\mu \mathrm{g}$ of tumor DNA and control DNA isolated from a human $\mathrm{T}$ cell line with the normal $c-m y c$ genomic structure (MOLT-16, a gift from Nino Neri) were digested with restriction enzyme PvuII, and processed as described (29).

$P C R$. The PCR was performed essentially as described (30). Primers included a c-myc oligomer of 20 residues homologous with nucleotides 1001 through 1020 (numbering in ref. 31) of the human c-mve gene and situated within the 1st exon downstream of the $\mathrm{P}_{2}$ promoter. This oligomer contained a $5^{\prime}$ linker sequence with sites for the restriction enzymes XhoI, SalI, and Cla-I, and had the nucleotide sequence: 5-GACTCGAGTCGACATCGATCGAAAACTTTGCCCATAGCA-3. A second c-myc primer was complementary to nucleotides 2859 through 2879 (31) and was situated within the 2 nd c-myc exon at its $5^{\prime}$ boundary. This oligomer contained a linker sequence with sites for the restriction enzymes EcoRI, SmaI, and HindIII, and had the nucleotide sequence: 5'-GAGAATTCCCGGGAAGCTTCCTGTTGGTGAAGCTAACGT-3'. Human $\beta$-actin primers included two 20 -mers. One primer was homologous with nucleotides 129 through 148 of the human $\beta$-actin $\mathrm{CDNA}(32)$ and had the nucleotide sequence: $5^{\prime}$-GTCTTCCCCTCCATCGTGGG-3'. The 2 nd $\beta$-actin primer was complementary to nucleotides 609 through 628 (32) and had the nucleotide sequence: 5'-CGCTCGGTGAGGATCTTCAT-3'.

\section{RESULTS}

Three of six medulloblastoma tumors analyzed have elevated $c$-myc expression. Initially, a series of seven pediatric tumors was examined for expression of genes that are indicative of differentiation and that are known to be expressed in cells of neuroectodermal origin. The tumors included five medulloblastomas, one ependymoma, and one neuroblastoma; a 6 th medulloblastoma specimen was collected later in the study. Using the selected cDNA probes, Northern analysis from these tumors indicated that only GFAP was expressed at detectable levels (Fig. 1, panel $a$, lanes 5-8). To assess the potential involvement of the c-myc protooncogene, we repeated the Northern blots with pmyc 413$\mathrm{R} / \mathrm{C}$, a cDNA that spans the 3rd exon of $c-m y c$ (Fig. 1, panel b). Two medulloblastomas (M-2 and M-3, Fig. 1, lanes 5 and 6 ) and the neuroblastoma (lane 10 ) were found to express high levels of c-myc mRNA when compared with adult rat brain and human fetal brain. In each case, the mRNA comigrated with cmyc mRNA from HeLa cells, which are wild type in size. A
Fig. 1. Northern blot analysis for GFAP and c-myc expression. Twenty $\mu \mathrm{g}$ of total cellular RNA were collected from the following tissue samples and loaded onto the indicated lanes. Lane 1, adult rat brain; lane 2, fetal human brain; lane 3, HeLa cells. Lanes 4 through 8 , RNA from medulloblastoma tumors as follows: lane 4, M-I; lane 5 , M-2; lane 6, M-3; lane 7, M-4; lane 8, M-5. Lane 9, ependymoma; lane 10, neuroblastoma. Transfer to Nytran filters (Schleicher \& Schuell, Keene, NH) and hybridization are as desdribed in Materials and Methods. The following oligolabeled probes were used: $a$ ) $\mathrm{Gl}$, a cDNA encoding GFAP, b) pmvc 4l3-R/C, a cDNA representing the entire 3rd exon of c-myc. Arrowheads with an $O$ mark the origins. Long arrows identify the $28 \mathrm{~S}$ and $18 \mathrm{~S}$ ribosomal markers.

comparison of band intensities in Figure 1 , panels $a$ and $b$, indicates an inverse correlation between GFAP expression and c-myc expression in the tumor samples analyzed in Figure 1, lanes 5 through 10 .

To control for any possible differences in the quantity of cellular RNA added to each lane, we analyzed by Northern assay the level of $\beta$-actin mRNA, which is relatively constant in different samples of similar tissue. The same Northern blot, containing RNA from the original five medulloblastoma tumors plus one additional medulloblastoma, was then stripped and exposed to the radiolabeled human c-myc probe, p $m y c$ 413-R/C (Fig. 2, panel $b$ ). Three of the medulloblastoma tumor samples ( $\mathrm{M}-2$, M-3, and M-6) exhibit detectable levels of c-myc message; long exposures did not reveal any $c-m y c$ expression in the remaining three tumor samples (M-1, M-4, and $\mathrm{M}-5$ ).

To confirm the results of the Northern blot assay and to investigate possible mechanisms of transcriptional deregulation, we examined the tumor $c-m y c$ mRNA by means of the ribonuclease protection assay. Identical aliquots of total cellular RNA from the six medulloblastomas in Figure 2 were hybridized with a radiolabeled antisense transcript of $m y c X-P s t$, which overlaps the junction of the $5^{\prime}$ end of the 2 nd exon and the 1 st intron of human $c-m y c$ (Fig. 3), digested with nuclease, and analyzed by gel electrophoresis. In Figure 3, protected fragments consistent with elevated c-myc mRNA expression in tumors $\mathrm{M}-2, \mathrm{M}-3$, and M- 6 were observed. The size of these bands was the same as obtained with HeLa c-myc mRNA, which is wild type in splicing, indicating that the tumor RNA were correctly spliced at the 2 nd exon $5^{\prime}$ boundary, and that intron sequences are absent from the mRNA. This observation argues against aberrant initiation 